\title{
Anticancer effect of YWHAZ silencing via inducing apoptosis and autophagy in gastric cancer cells
}

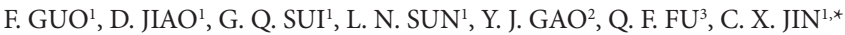 \\ ${ }^{1}$ Department of Ultrasound, China-Japan Union Hospital of Jilin University, Changchun 130033, People's Republic of China; ${ }^{2}$ Department of \\ Gastrointestinal Surgery, China-Japan Union Hospital of Jilin University, Changchun 130033, People's Republic of China; ${ }^{3}$ Jilin Provincial Key \\ Laboratory of Surgical Translational Medicine, Division of Thyroid Surgery, China-Japan Union Hospital of Jilin University, Changchun 130033, \\ People's Republic of China \\ ${ }^{\star}$ Correspondence: jinchunxiang7777@126.com
}

Received September 22, 2017 / Accepted January 5, 2018

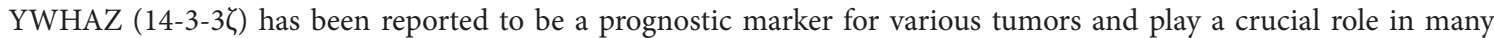
oncogenic processes, including proliferation, migration and invasion. However, the functional role and mechanism of YWHAZ in gastric cancer (GC) are not detailed and still require study. Herein, the endogenous expression of YWHAZ in gastric cancer cell line BGC-823 was silenced by YWHAZ-specific short hairpin RNA (shRNA). Our data showed that YWHAZ silencing resulted in cell cycle arrest in BGC-823 cells. Further, YWHAZ-silenced BGC-823 cells acquired increased apoptosis rate, which was confirmed by increased levels of cleaved caspase-3, cleaved PARP and Bax and decreased the Bcl-2 level. Suppression of YWHAZ also promoted autophagy, confirmed by the up-regulation of LC3II /LC3I ratio, and downregulation of p62 level. Moreover, YWHAZ suppression inhibited the activation of PI3K/AKT/mTOR signaling pathway in BGC-823 cells. LY294002 (PI3K/AKT inhibitor, $200 \mathrm{nM}$ ) further promoted YWHAZ silencing-induced apoptosis and autophagy in BGC-823 cells while insulin-like growth factor-1 (IGF-1; PI3K/AKT agonist, $10 \mathrm{ng} / \mathrm{ml}$ ) had the opposite effect. Finally, suppression of YWHAZ inhibited the growth of the xenograft tumor in vivo. This study provides extended evidence that YWHAZ can be a potential therapeutic target for GC.
\end{abstract}

Key words: YWHAZ, gastric cancer, apoptosis, autophagy, PI3K/AKT/mTOR

Gastric cancer (GC) ranks as the third leading cause of cancer mortality worldwide in 2012 [1]. Its incidence and mortality in East Asia are higher than other global regions worldwide, and GC is confirmed to be the second most deadly cancer in China [2]. Despite significant advances in preventive and diagnostic techniques, GC patients are often diagnosed at a late stage and the prognosis remains poor [3]. It is therefore essential to identify and develop novel therapeutic targets for early GC diagnosis and treatment.

YWHAZ, also called $14-3-3 \zeta$, is an important member of the highly conserved 14-3-3 family that plays key roles in many biological processes in eukaryotic cells [4-6]. Previous study has shown that YWHAZ takes part in the regulation of neurogenesis and neural progenitor differentiation [7], and YWHAZ not only participates in the regulation of many physiological activities, but it is also a novel molecular target and prognostic marker for various cancers $[4,8,9]$. YWHAZ has been demonstrated to be over-expressed in several cancers, including GC, pancreatic cancer, and intrahepatic cholangiocarcinoma where it promoted cancer initiation and progression [9-12]. Moreover, over-expression of YWHAZ contributed to the proliferation and survival of both GC and prostate cancer cells [13, 14]. MircoRNA-375 performed proapoptotic function in GC cells through down-regulating the YWHAZ level [15]. Although several studies have investigated the regulatory roles of YWHAZ in various cancer cells, the detailed mechanisms of YWHAZ in GC still remain unclear and require further elucidation.

Herein, specific YWHAZ-shRNA was applied to silence the expression of YWHAZ in GC cells, and the effects of YWHZA silencing on cell apoptosis, autophagy and related mechanisms were assessed in in vitro GC cells for the first time. We also detected the effect of YWHAZ on the growth of tumor xenograft in nude mice in vivo.

\section{Materials and methods}

Cell line and culture. BGC-823 cell line was purchased from Zhong Qiao Xin Zhou Biotechnology Co., Ltd. (Shanghai, China). The cells were cultured in RPMI-1640 
medium (Gibco, USA) supplemented with $10 \%$ FBS (Hyclone, USA) at $37^{\circ} \mathrm{C}$ in a $5 \% \mathrm{CO}_{2}$ incubator.

Plasmids construction and stable cell lines generation. The shRNA for human YWHAZ gene (5'-GATCCCCGCTGAGCGATATGATGACATTCAAGAGATGTCATCATATCGCTCAGCTTTTT-3') and the negative control (NC) shRNA (5'-GATCCCCTTCTCCGAACGTGTCACGTTTCAAGAGAACGTGACACGTTCGGAGAATTTTT-3') were inserted into the pRNA-H1.1 vector. pRNA-H1.1-YWHAZ shRNA or pRNA-H1.1-NC shRNA was transfected into BGC-823 cells by lipofectamine 2000 (Invitrogen, USA) according to the manufacturer's instructions. Stable YWHAZ-silenced BGC-823 cells were selected by incubation with $800 \mu \mathrm{g} / \mathrm{ml} \mathrm{G418}$ at 24 hours after transfection. The interference efficiency was verified by real-time PCR and western blot, as described below.

Quantitative real-time PCR (qPCR). The mRNA expression of YWHAZ in BGC-823 cells was detected by qPCR. Total RNA was extracted from cells with RNApure total RNA fast isolation kit (BioTeke, Beijing, China) and cDNA was obtained by Super M-MLV reverse transcriptase (BioTeke, Beijing, China) following the manufacturer's instructions. The qPCR was conducted in $20 \mu \mathrm{l}$ volume on Exicycler 96 (BIONEER, Daejeon, Korea). The primer sequences used were synthesized by Sangon Biotech Co., Ltd (Shanghai, China) and presented as follows: YWHAZ (forward-5'TGTTGTAGGAGCCCGTAG-3', reverse-5'-GCAACCTCAGCCAAGTAA-3') and $\beta$-actin (forward-5'-CTTAGTTGCGTTACACCCTTTCTTG-3', reverse-5'-CTGTCACCTTCACCGTTCCAGTTT- $3^{\prime}$ ). $\beta$-actin was used as the internal control and mRNA expression was calculated by the $2^{-\Delta \Delta C T}$ method.

Western blot. BGC-823 cells were lysed by RIPA buffer (Beyotime, China) supplemented with $1 \%$ phenylmethylsulfonyl fluoride (PMSF; Beyotime). The protein concentration was determined by BCA Protein Assay Kit (Beyotime) according to the manufacturer's instructions. Equal amounts of protein samples were then subjected to sodium dodecyl sulfate polyacrylamide gel electrophoresis (SDS-PAGE) and transferred to PVDF membrane. After blocking with 5\% skim milk for 1 hour, the membrane was incubated at $4{ }^{\circ} \mathrm{C}$ overnight with primary antibodies YWHAZ (1:500, Sangon Biotech, China), cleaved caspase-3 (1:500) and cleaved PARP (1:500, Cell Signaling Technology, USA), Bcl-2 (1:500, Sangon Biotech, China), Bax (1:500, Sangon Biotech, China), LC3 (1:500) and p62 (1:500, Cell Signaling Technology, USA), p-PI3K (1:1000) and PI3K (1:500, Abcam, UK), p-AKT (1:500), AKT (1:500), p-Mtor (1:500) and mTOR (1:500, Cell Signaling Technology, USA), $\beta$-actin (1:500, Bioss, China). The membrane was then incubated with HRP-conjugated goat anti-rabbit or goat anti-mouse secondary antibodies at $37^{\circ} \mathrm{C}$ for 45 minutes. The blots were visualized with ECL reagent (Beyotime, China).

Cell apoptosis analysis by flow cytometry. The apoptosis of BGC-823 cells was detected by Annexin V-FITC/PI double staining Apoptosis Assay Kit according to the manufacturer's instructions (KeyGEN, China). Briefly, cells were collected and re-suspended with $500 \mu \mathrm{l}$ Binding Buffer and singlecell suspensions were incubated with $5 \mu \mathrm{l}$ Annexin V-FITC and $5 \mu \mathrm{l}$ PI for 15 minutes in the dark. Cell apoptosis was analyzed on a flow cytometer (BD, USA).

Cell cycle analysis by flow cytometry. The cell cycle was analysed by Cell Cycle and Apoptosis Analysis Kit (Beyotime, China) according to the manufacturer's instructions. Briefly, cells were collected and fixed with pre-cooling $70 \%$ ethanol at $4{ }^{\circ} \mathrm{C}$ for 2 hours. The fixed cells were washed twice with PBS, re-suspended with $500 \mu \mathrm{l}$ staining buffer, then incubated with $25 \mu \mathrm{lI}$ and $10 \mu \mathrm{l}$ RNase $\mathrm{A}$ at $37^{\circ} \mathrm{C}$ for 30 minutes away from light. The cell cycle was then detected by flow cytometry (BD, USA).

In vivo tumor xenograft study. Healthy female nude $\mathrm{BALB} / \mathrm{c}$ mice (4-weeks-old) were purchased from HFK Bioscience Co.,LTD (Beijing, China). $1 \times 10^{7}$ BGC-823 cells in $100 \mu \mathrm{l}$ serum free PRMI 1640 from parental, NC, and YWHAZ silencing groups were subcutaneously injected into the fossa axillaris of nude mice ( $\mathrm{n}=6$ for each group). The tumors were measured and the volume was calculated every three days after tumor nodules were observed. The mice were sacrificed 28 days after the injection, and the tumors were removed, weighed and collected. The animals were cared for in accordance with the principles and guidelines for the Care and Use of Laboratory Animals. All experimental procedures performed in studies involving animals were in accordance with the Institutional Animal Care and Use Committee guidelines of Jilin University.

Statistical analysis. All experimental values were expressed as mean \pm standard deviation (SD). The Student's $\mathrm{t}$ test compared the differences between the two groups, and p-value less than 0.05 was statistically significant.

\section{Results}

YWHAZ expression in gastric cancer cells. The protein levels of YWHAZ in four GC cell lines (BGC-823, SGC-7901, HGC-27 and MGC-803) were assessed by western blot, and the result suggested that the level of YWHAZ in BGC-823 cells was higher than in other three cells lines (Figure 1A). Hence, the BGC-823 cell line was chosen to establish the stable YWHAZ silencing GC cell line. YWHAZ-shRNA was applied to inhibit the endogenous expression of YWHAZ. Figures $1 \mathrm{~B} \& \mathrm{C}$ show that both $\mathrm{mRNA}$ and protein expressions of YWHAZ were significantly decreased in YWHAZshRNA transfected BGC-823 cells.

YWHAZ suppression induced cell cycle arrest and apoptosis in BGC-823 cells. Figure 2A highlights the determination of cell cycle progression by flow cytometry. Suppression of YWHAZ resulted in significant decrease in the number of cells in BGC-823 cells' $S$ phase compared to the NC group. Although YWHAZ silencing increased the number of cells in the G1 phase, there was no signifi- 
A
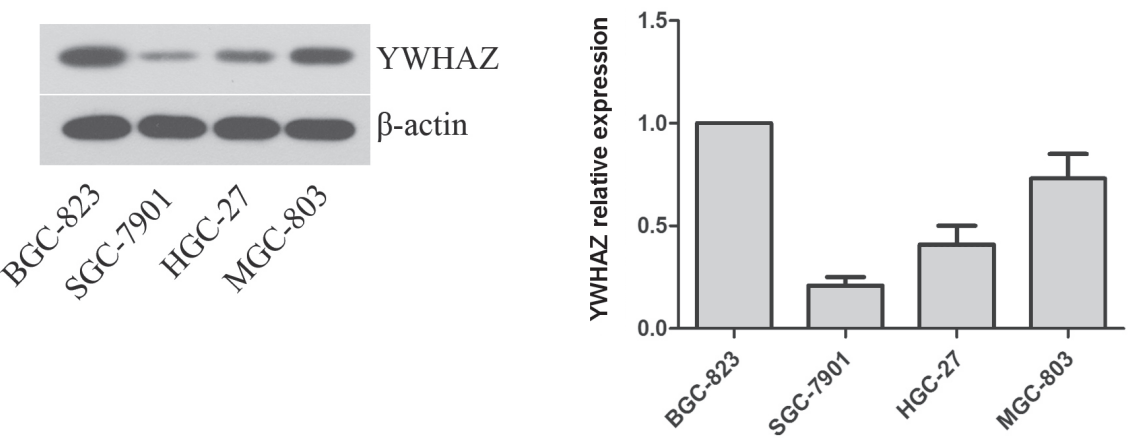

$\mathrm{B}$

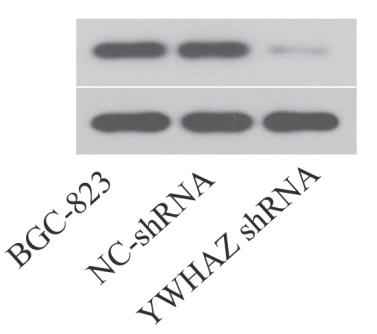

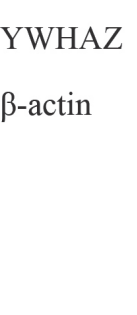
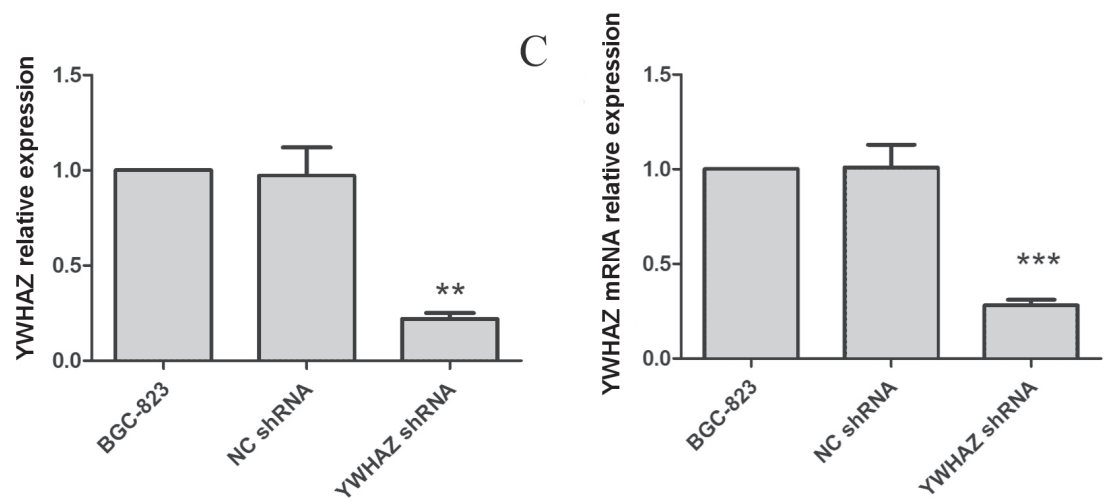

Figure 1. Establishment of stable YWHAZ-silenced cells. A) Western blot analysis evaluated the YWHAZ protein level in various GC cell lines and $\beta$-actin was used as a loading control. B) The YWHAZ protein level after transfection of YWHAZ-shRNA in BGC-823 cells was detected by western blot. C) YWHAZ mRNA expression after transfection of YWHAZ-shRNA in BGC-823 cells was detected by qPCR. The results are confirmed in three repeated experiments. Each value is shown as the mean $\pm S D(n=3) .{ }^{* *} p<0.01,{ }^{* * *} p<0.001$, versus the NC group.

cant statistical difference. In addition, the apoptosis rate in BGC-823 cells was markedly enhanced by YWHAZ silencing compared with the NC group (Figure 2B). Figures $2 \mathrm{C}-\mathrm{G}$ show apoptosis-related proteins levels determined by western blot, where YWHAZ suppression significantly increased protein levels in pro-apoptotic proteins cleaved caspase-3, cleaved PARP and Bax, and obviously decreased the level of antiapoptotic protein $\mathrm{Bcl}-2$ in BGC-823 cells.

Suppression of YWHAZ promoted autophagy in BGC-823 cells. The LC3 and p62 autophagy-related protein levels were detected in order to evaluate the effect of YWHAZ on autophagy. Figure 3 reveals that YWHAZ suppression increased the ratio of LC3 II/LC3 I and decreased the level of p62 in BGC-823 cells, thus indicating that YWHAZ silencing promoted autophagy in the BGC-823 cells.

The PI3K/AKT/mTOR signaling pathway is involved in the regulation of apoptosis and autophagy by YWHAZ suppression. To further evaluate the detailed mechanism of YWHAZ in BGC-823 cells, the canonical PI3K/AKT/mTOR signaling pathway was selected. Figures $4 \mathrm{~A}-\mathrm{C}$ show that the protein levels of phosphorylated PI3K, AKT and mTOR were markedly decreased by suppression of YWHAZ in BGC-823 cells while the total PI3K, AKT and mTOR had no significant changes. The PI3K/AKT/mTOR pathway role was elucidated by YWHAZ silenced BGC-823 cells treated with LY294002 or IGF-1; the respective inhibitor and activator of the PI3K/ AKT pathway. As shown in Figure 4D, LY294002 treatment significantly enhanced YWHAZ silencing-induced apoptosis in BGC-823 cells, while IGF-1 treatment inhibited apoptosis.

While LY294002 treatment up-regulated the ratio of LC3II /LC3I and down-regulated the level of p62 in YWHAZsilenced BGC-823 cells, IGF-1 treatment had the opposite effect. These results suggest that PI3K/AKT/mTOR signaling pathway is involved in YWHAZ mechanisms in BGC-823 cells.

Suppression of YWHAZ inhibited the growth of xenograft tumors in vivo. The nude mouse tumorigenesis test assessed the effect of YWHAZ suppression on in vivo. tumorigenesis ability. Figures $5 \mathrm{~A}$ and $\mathrm{B}$ highlight that the volume of xenograft tumor generated by YWHAZ-silenced BGC-823 cells was significant less than that generated by NC BGC- 823 cells. The xenograft tumor weight was also significantly reduced when YWHAZ was silenced (Figure 5C) and figure $5 \mathrm{D}$ validates the protein level of YWHAZ in tumor tissues. 
A

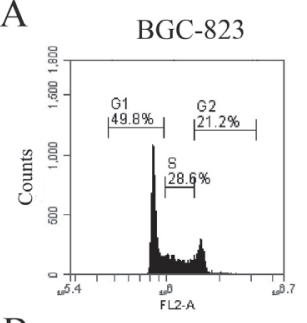

$\mathrm{B}$

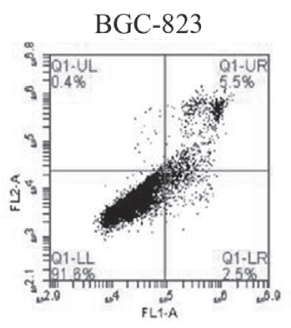

$\mathrm{C}$

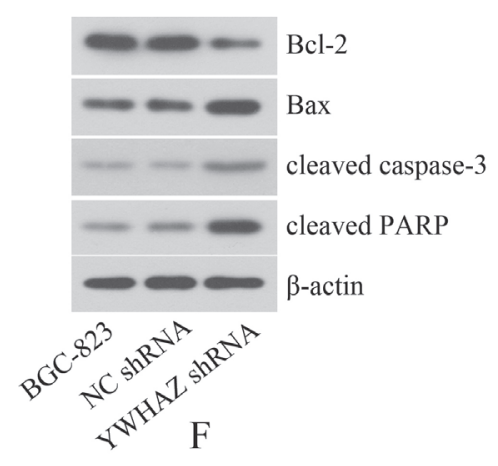

D

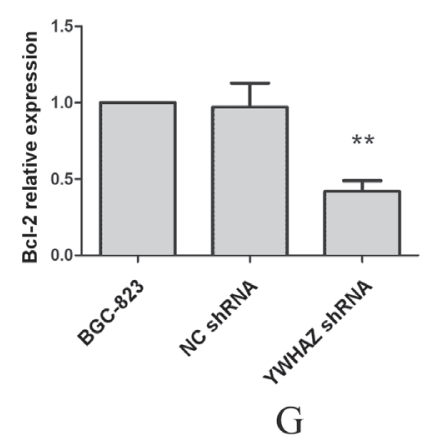

YWHAZ shRNA

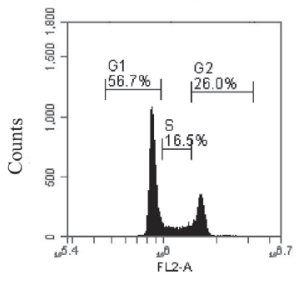

YWHAZ shRNA

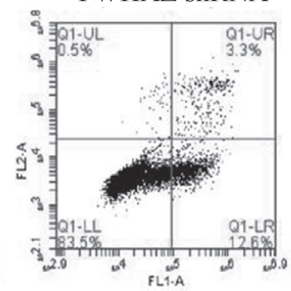

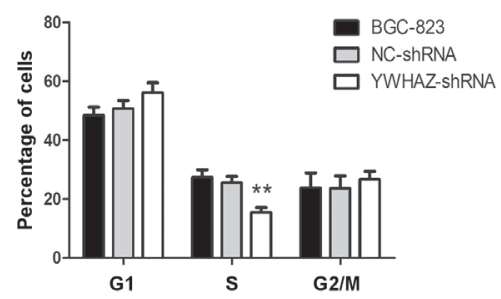

$\mathrm{E}$
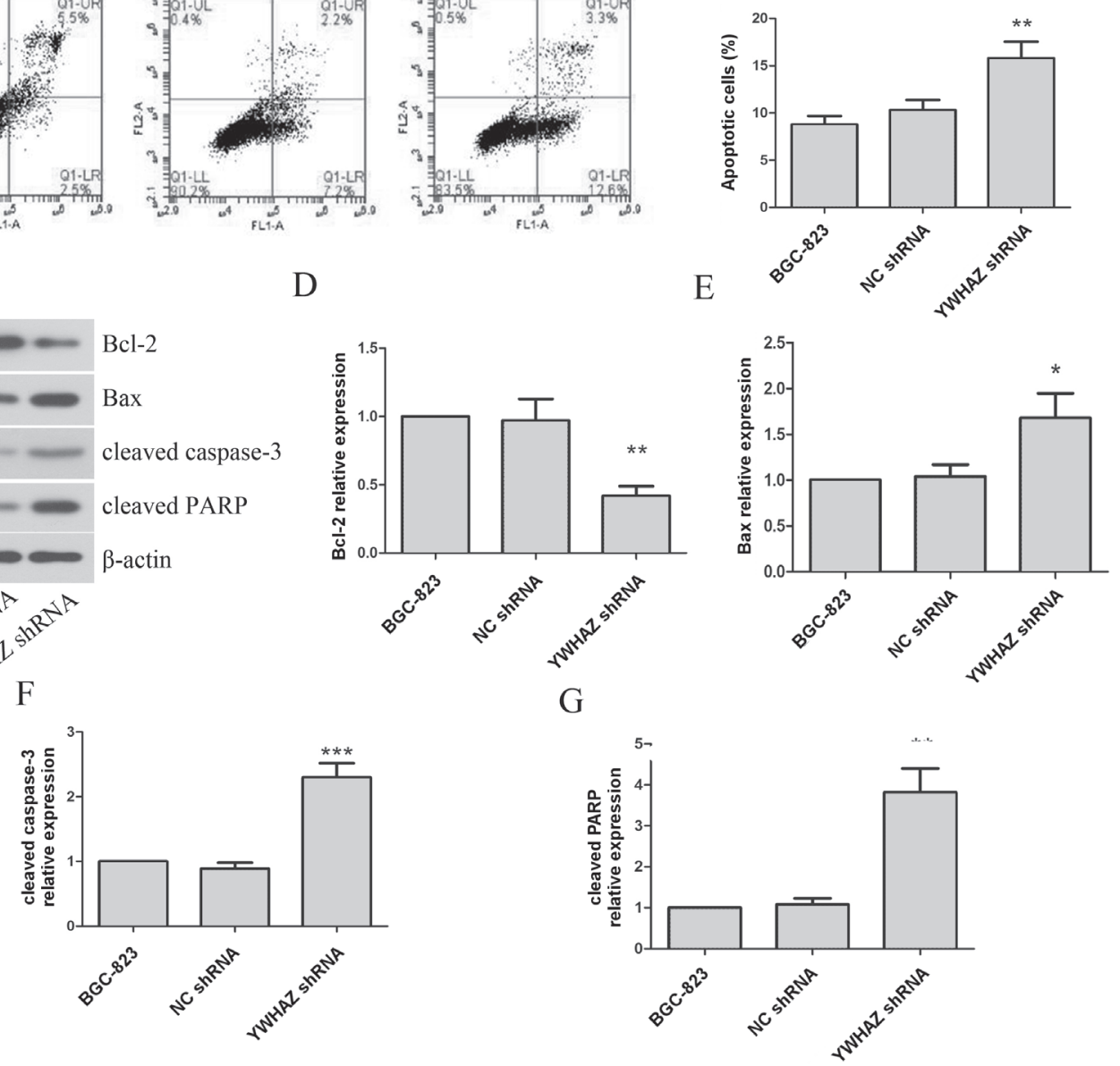

Figure 2. The effect of YWHAZ suppression on cell cycle and apoptosis in BGC-823 cells. A) Cell cycle progression in BGC-823 cells was detected by flow cytometry. The percentage of cells in each cell cycle phase is shown. B) Annexin V/PI staining was used to detect cell apoptosis in BGC-823 cells. The percentage of apoptosis cells is shown. C) The protein levels of Bcl-2, Bax, cleaved caspase-3, and cleaved PARP in BGC-823 cells were determined by western blot analysis, with $\beta$-actin used as the loading control. D-G) Gray scanning analysis of the protein bands. The results are confirmed in three repeated experiments. Each value is shown as mean $\pm \mathrm{SD}(\mathrm{n}=3) .{ }^{*} \mathrm{p}<0.05,{ }^{* *} \mathrm{p}<0.01,{ }^{* *} \mathrm{p}<0.001$, versus the $\mathrm{NC}$ group.

\section{Discussion}

GC is always diagnosed at advanced stages, leading to poor prognosis for most patients with GC [16]. Therefore, searching for promising molecular target for early diagnosis and treatment is essential for GC patients, and YWHAZ, as a member of 14-3-3 family, is known to participate in multiple cellular processes. A previous study demonstrated that YWHAZ was frequently over-expressed in GC tumor tissues, and this significantly correlated with malignancy in GC patients. Silencing YWHAZ inhibited proliferation, migration and invasion of GC cells [13]. The novelty of this study is that we fully investigated the role of YWHAZ in apoptoisis, autophagy and its related mechanisms in GC cells in vitro and tumorigenesis in vivo. Our data showed that suppression of YWHAZ promoted GC cell apoptosis and autophagy by inactivating the PI3K/AKT/mTOR 
A

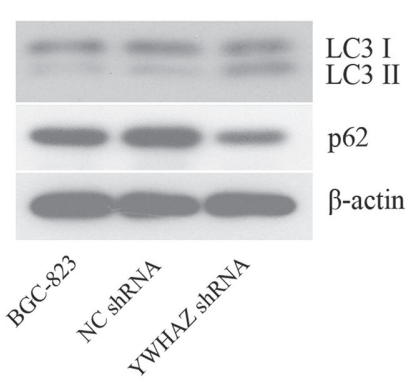

$\mathrm{B}$

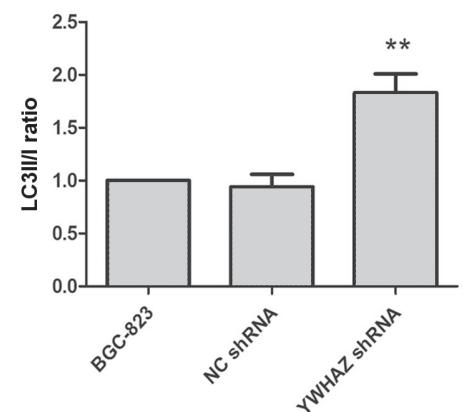

$\mathrm{C}$

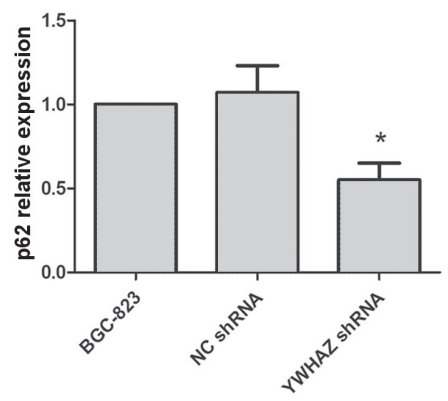

Figure 3. The effect of YWHAZ suppression on autophagy in BGC-823 cells. A) The protein levels of LC3 and p62 were assessed by western blot. $B-C)$ Gray scanning analysis of the protein bands. The results are confirmed in three repeated experiments. Each value is shown as mean \pm SD $(n=3)$. ${ }^{\star} \mathrm{p}<0.05,{ }^{* *} \mathrm{p}<0.01$, versus the NC group.

A

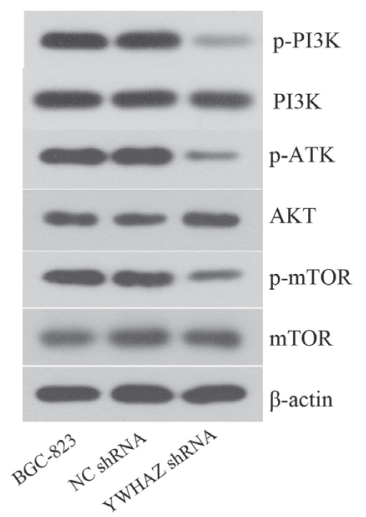

B

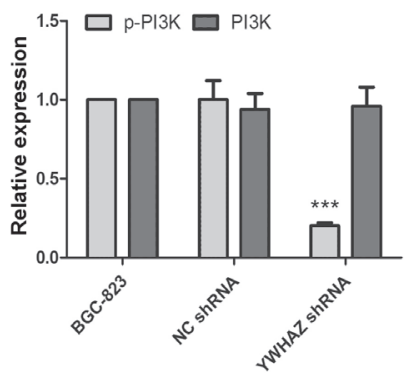

C

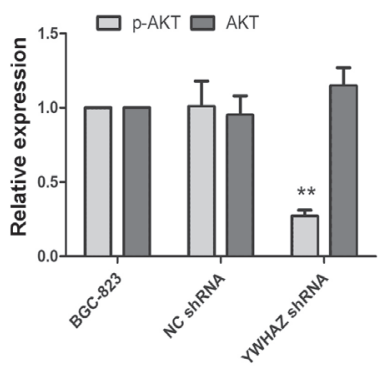

D

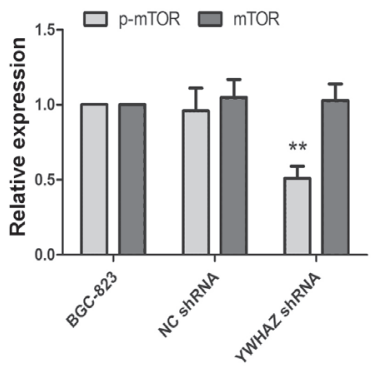

E

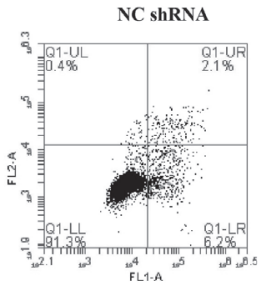

F

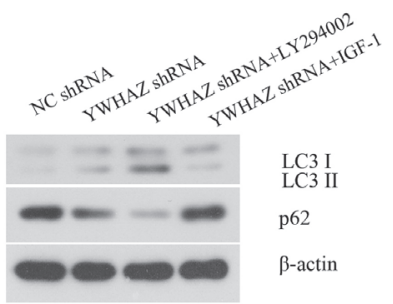

YWHAZ ShRNAZ

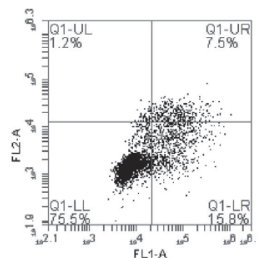

G

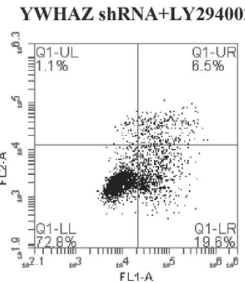

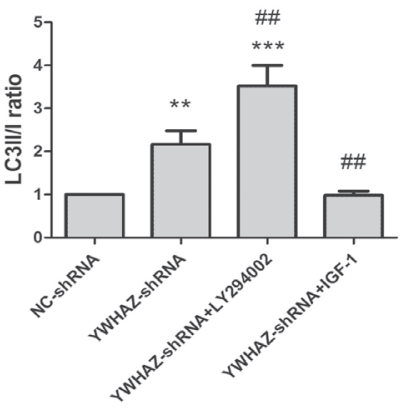

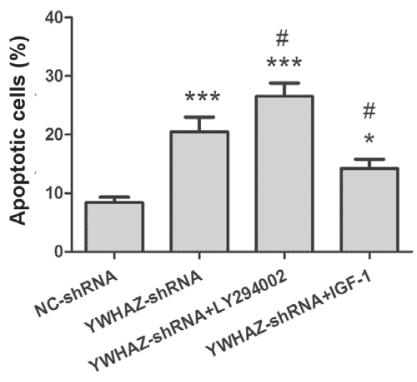

$\mathrm{H}$

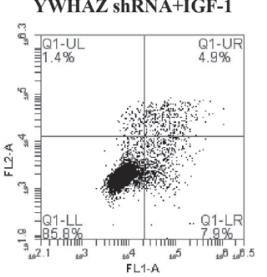

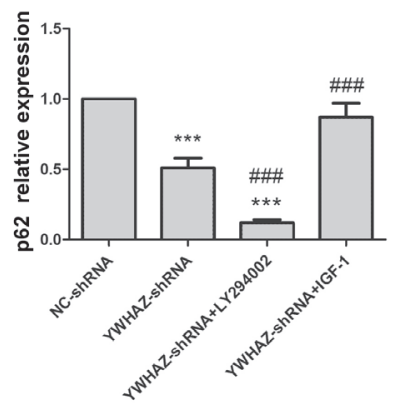

Figure 4. The PI3K/AKT/mTOR signaling pathway is involved in YWHAZ suppression in BGC-823 cells. The protein levels of p-PI3K, PI3K, p-AKT, AKT, p-mTOR, mTOR in BGC-823 cells were assessed by western blot. B-D) Gray scanning analysis of the protein bands. E) YWHAZ-silenced BGC823 cells were treated with $200 \mathrm{nM}$ LY294002 or $10 \mathrm{ng} / \mathrm{ml} \mathrm{IGF-1} \mathrm{for} 12 \mathrm{~h}$. The apoptosis of BGC-823 cells in different treatment groups was evaluated by Annexin V/PI staining. The percentage of apoptosis cells is shown. F) YWHAZ-silenced BGC-823 cells were treated with $200 \mathrm{nM} \mathrm{LY294002} \mathrm{or} 10 \mathrm{ng} /$ $\mathrm{ml} \mathrm{IGF-1} \mathrm{for} 12 \mathrm{~h}$ and LC3 and p62 protein levels were assessed by western blot. G-H) Gray scanning analysis of the protein bands. The results are confirmed in three repeated experiments. Each value is shown as mean $\pm \operatorname{SD}(n=3) .{ }^{*} p<0.05,{ }^{* *} p<0.01,{ }^{* * *} p<0.001$, versus the $N C$ group. ${ }^{*} p<0.05$, ${ }^{* *} p<0.01$, ${ }^{\# \# * *} \mathbf{p}<0.001$, versus the YWHAZ-shRNA group. 
A
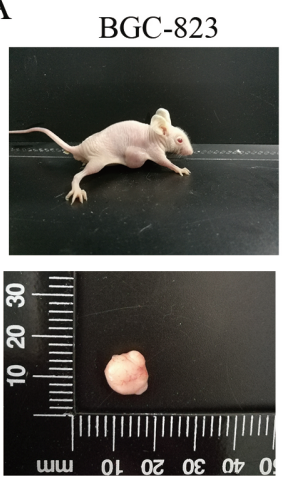

$\mathrm{C}$
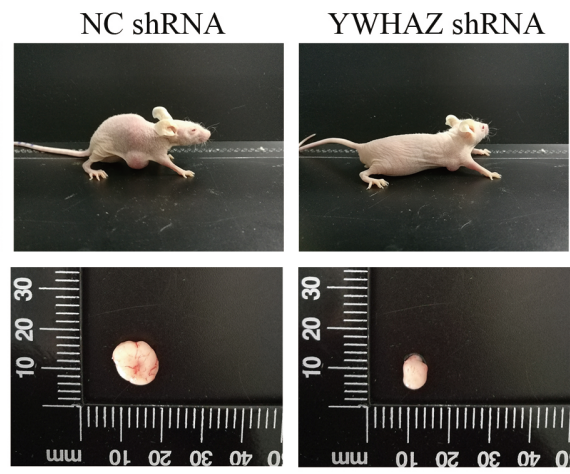

B

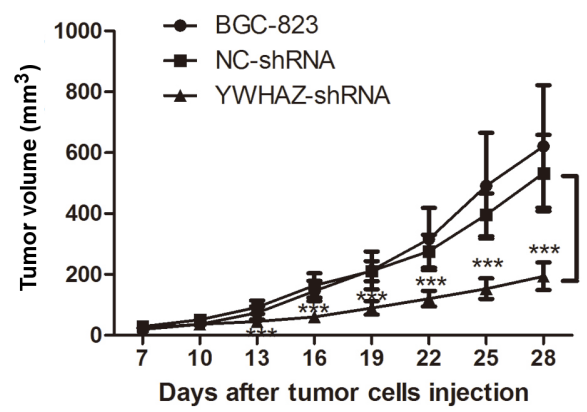

$\mathrm{D}$

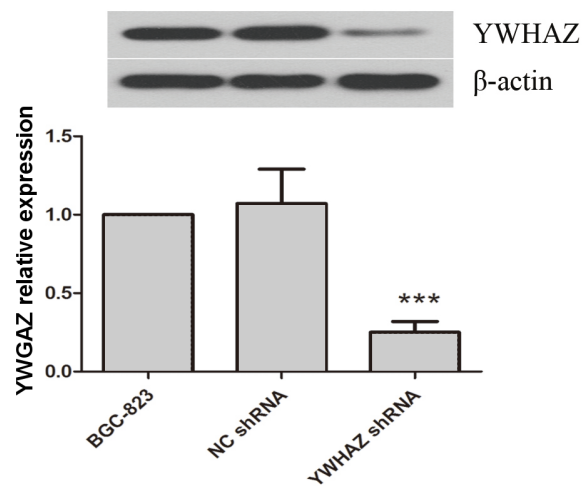

Figure 5. In vivo suppression of YWHAZ inhibited tumorigenesis of GC. A) Images of nude mice and removed xenograft tumors at 28 days after the injection of YWHAZ-silenced or NC BGC-823 cells. B) The tumor growth-curve of tumor volume is shown. C) The tumor weight of different groups was presented. D) The YWHAZ protein level in tumor tissues was measured by western blot. The results are confirmed in three repeated experiments. Each value is shown as mean $\pm \mathrm{SD}(n=6) .{ }^{* *} \mathrm{p}<0.001$ compared to the NC group.

signaling pathway and that YWHAZ suppression reduced tumorigenic ability in vivo.

The programmed cell death in apoptosis is cell suicide, and this has key roles in multiple physiological and pathological processes, which is an important factor for selfstabilization. The occurrence of tumor is largely attributable to abnormal cell apoptosis. Apoptosis has been the main target and method in tumor therapy so far and regulation of apoptosis-related gene expression is the key method used to induce tumor cell apoptosis.

Here, the Bcl-2 family proteins play important roles in the regulation of apoptosis [17]. Anti-apoptotic Bcl-2 and pro-apoptotic Bax proteins are two crucial members of the Bcl-2 family. The ratio of Bcl-2/Bax closely correlates with cancer cell apoptosis and this is considered a predictive marker $[18,19]$. Caspase-3 is a key effector molecule in the apoptotic signal transduction pathway [20], where activation cleaves its PARP substrate resulting in loss of DNA repair function and apoptosis [21]. Our results confirm that suppression of YWHAZ in BGC-823 cells significantly induced apoptosis by down-regulating the $\mathrm{Bcl}-2$ level and up-regulating Bax, cleaved caspase-3 and the cleaved PARP levels.
Autophagy ensures that cellular components are orderly degraded and recycled in eukaryotic cells. The specific LC3 marker for autophagy has two important subtypes, LC3-I and LC3-II, and autophagous activation can be demonstrated by the conversion of LC3-I to LC3-II [22]. P62 is another important autophagy-related protein and this can be degraded in autophagosomes during the autophagy process. Thus, the level of p62 level inversely correlates with autophagy level [23]. Autophagy has complicated effects in different conditions; although it is an adaptive response which protects cells against injury it can also mediate programmed cell death.

Based on this dual mechanism, autophagy has been demonstrated to play contradictory roles in different cancer stages. A recent study showed that activation of dopamine receptor D5 suppressed GC cells by inducing autophagic cell death [24]. More recent reports demonstrated that induction of autophagy inhibited GC cells proliferation [25-27]. However, research has also suggested that silencing AQP3 induces GC cell apoptosis by inhibiting autophagy [28]. Herein, suppression of YWHAZ significantly induced autophagy in BGC-823 cells; clearly shown by increased LC3II /LC3I ratio and decreased p62 level. These results 
indicate that YWHAZ silencing inhibited GC progression by inducing autophagous cell death.

The PI3K/AKT/mTOR signaling pathway is a canonical intracellular signal transduction pathway, and it has been reported to be involved in GC cell regulation of proliferation, apoptosis and invasion [29-31]. This pathway also plays crucial roles in autophagy regulation. A recent study suggested that IL-37 induces autophagy in liver cancer by inhibiting the PI3K/AKT/mTOR pathway [32]. In addition, Ding et al showed that tanshinone IIA suppressed proliferation and induced autophagy of U251 cells by inhibiting the $\mathrm{PI} 3 \mathrm{~K} / \mathrm{AKT} / \mathrm{mTOR}$ pathway[33].

Finally, our results are quite consistent with previous studies, and they clearly confirm that YWHAZ-silencing induces apoptosis and autophagy in BGC-823 cells by inactivation of the PI3K/AKT/mTOR pathway. Moreover, silencing YWHAZ restrained tumorigenesis in nude mice in vivo; and we therefore suggest YWHAZ is a potential therapeutic target in Gastric Cancer

\section{References}

[1] FERLAY J, SOERJOMATARAM I, DIKSHIT R, ESER S, MATHERS $C$ et al. Cancer incidence and mortality worldwide: sources, methods and major patterns in GLOBOCAN 2012. Int J Cancer 2015; 136: E359-386. https://doi. org/10.1002/ijc.29210

[2] CHEN W, ZHENG R, BAADE PD, ZHANG S, ZENG H et al. Cancer statistics in China, 2015. CA Cancer J Clin 2016; 66: 115-132. https://doi.org/10.3322/caac.21338

[3] KAPADIA CR. Gastric atrophy, metaplasia, and dysplasia: a clinical perspective. J Clin Gastroenterol 2003; 36: S29-36.

[4] MATTA A, SIU KM AND RALHAN R. 14-3-3 zeta as novel molecular target for cancer therapy. Expert Opin Ther Targets 2012; 16: 515-523. https://doi.org/10.1517/14728222.20 12.668185

[5] AITKEN A. Post-translational modification of 14-3-3 isoforms and regulation of cellular function. Semin Cell Dev Biol 2011; 22: 673-680. https://doi.org/10.1016/j. semcdb.2011.08.003

[6] YANG X, LEE WH, SOBOTT F, PAPAGRIGORIOU E, ROBINSON CV et al. Structural basis for protein-protein interactions in the 14-3-3 protein family. Proc Natl Acad Sci U S A 2006; 103: 17237-17242. https://doi.org/10.1073/ pnas.0605779103

[7] TOYO-OKA K, WACHI T, HUNT RF, BARABAN SC, TAYA $S$ et al. 14-3-3epsilon and zeta regulate neurogenesis and differentiation of neuronal progenitor cells in the developing brain. J Neurosci 2014; 34: 12168-12181. https://doi. org/10.1523/JNEUROSCI.2513-13.2014

[8] TZIVION G, GUPTA VS, KAPLUN L, BALAN V. 14-3-3 proteins as potential oncogenes. Semin Cancer Biol 2006; 16: 203-213. https://doi.org/10.1016/j.semcancer.2006.03.004

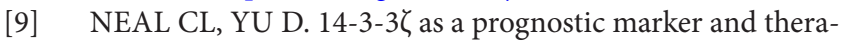
peutic target for cancer. Expert Opin Ther Targets 2010; 14: 1343-1354. https://doi.org/10.1517/14728222.2010.531011
[10] JANG JS, CHO HY, LEE YJ, HA WS, KIM HW. The differential proteome profile of stomach cancer: identification of the biomarker candidates. Oncol Res 2004; 14: 491-499.

[11] KLEMM C, DOMMISCH H, GOKE F, KREPPEL M, JEPSEN S et al. Expression profiles for 14-3-3 zeta and CCL20 in pancreatic cancer and chronic pancreatitis. Pathol Res Pract 2014; 210: 335-341. https://doi.org/10.1016/j. prp.2014.01.001

[12] ZHANG C, LIU LX, DONG ZR, SHI GM, CAI JB et al. Up-regulation of 14-3-3zeta expression in intrahepatic cholangiocarcinoma and its clinical implications. Tumour Biol 2015; 36: 1781-1789. https://doi.org/10.1007/s13277-0142780-5

[13] NISHIMURA Y, KOMATSU S, ICHIKAWA D, NAGATA H, HIRAJIMA $S$ et al. Overexpression of YWHAZ relates to tumor cell proliferation and malignant outcome of gastric carcinoma. Br J Cancer 2013; 108: 1324-1331. https://doi. org/10.1038/bjc.2013.65

[14] MURATA T, TAKAYAMA K, URANO T, FUJIMURA T, ASHIKARI D et al. 14-3-3zeta, a novel androgen-responsive gene, is upregulated in prostate cancer and promotes prostate cancer cell proliferation and survival. Clin Cancer Res 2012; 18: 5617-5627. https://doi.org/10.1158/1078-0432. CCR-12-0281

[15] TSUKAMOTO Y, NAKADA C, NOGUCHI T, TANIGAWA M, NGUYEN LT et al. MicroRNA-375 is downregulated in gastric carcinomas and regulates cell survival by targeting PDK1 and 14-3-3zeta. Cancer Res 2010; 70: 2339-2349. https://doi.org/10.1158/0008-5472.CAN-09-2777

[16] SONG WC, QIAO XL, GAO XZ. A comparison of endoscopic submucosal dissection (ESD) and radical surgery for early gastric cancer: a retrospective study. World J Surg Oncol 2015; 13: 309. https://doi.org/10.1186/s12957-015-0724-1

[17] EDLICH F. BCL-2 proteins and apoptosis: Recent insights and unknowns. Biochem Biophys Res Commun 2017. https://doi.org/10.1016/j.bbrc.2017.06.190

[18] SCOPA CD, VAGIANOS C, KARDAMAKIS D, KOURELIS TG, KALOFONOS HP et al. bcl-2/bax ratio as a predictive marker for therapeutic response to radiotherapy in patients with rectal cancer. Appl Immunohistochem Mol Morphol 2001; 9: 329-334.

[19] CHRESTA CM, MASTERS JR, HICKMAN JA. Hypersensitivity of human testicular tumors to etoposide-induced apoptosis is associated with functional p53 and a high Bax:Bcl-2 ratio. Cancer Res 1996; 56: 1834-1841.

[20] PORTER AG, JANICKE RU. Emerging roles of caspase-3 in apoptosis. Cell Death Differ 1999; 6: 99-104. https://doi. org/10.1038/sj.cdd.4400476

[21] LI X, DARZYNKIEWICZ Z. Cleavage of Poly(ADP-ribose) polymerase measured in situ in individual cells: relationship to DNA fragmentation and cell cycle position during apoptosis. Exp Cell Res 2000; 255: 125-132. https://doi. org/10.1006/excr.1999.4796

[22] KABEYA Y, MIZUSHIMA N, UENO T, YAMAMOTO A, KIRISAKO $\mathrm{T}$ et al. LC3, a mammalian homologue of yeast Apg8p, is localized in autophagosome membranes after processing. EMBO J 2000; 19: 5720-5728. https://doi. org/10.1093/emboj/19.21.5720 
[23] MATHEW R, KARP CM, BEAUDOIN B, VUONG N, $\mathrm{CHEN} \mathrm{G}$ et al. Autophagy suppresses tumorigenesis through elimination of p62. Cell 2009; 137: 1062-1075. https://doi. org/10.1016/j.cell.2009.03.048

[24] LENG ZG, LIN SJ, WU ZR, GUO YH, CAI L et al. Activation of DRD5 (dopamine receptor D5) inhibits tumor growth by autophagic cell death. Autophagy 2017; 13: 14041419. https://doi.org/10.1080/15548627.2017.1328347

[25] ZHAO E, TANG C, JIANG X, WENG X, ZHONG X et al. Inhibition of cell proliferation and induction of autophagy by KDM2B/FBXL10 knockdown in gastric cancer cells. Cell Signal 2017; 36: 222-229. https://doi.org/10.1016/j.cellsig.2017.05.011

[26] LI C, WANG Y, WANG C, YI X, LI M et al. Anticancer activities of harmine by inducing a pro-death autophagy and apoptosis in human gastric cancer cells. Phytomedicine 2017; 28: 10-18. https://doi.org/10.1016/j.phymed.2017.02.008

[27] LV LX, ZHOU ZX, ZHOU Z, ZHANG LJ, YAN R et al. Hispidin induces autophagic and necrotic death in SGC-7901 gastric cancer cells through lysosomal membrane permeabilization by inhibiting tubulin polymerization. Oncotarget 2017; 8: 26992-27006. https://doi.org/10.18632/oncotarget. 15935

[28] CHEN L, LI Z, ZHANG Q, WEI S, LI B et al. Silencing of AQP3 induces apoptosis of gastric cancer cells via downregulation of glycerol intake and downstream inhibition of lipogenesis and autophagy. Onco Targets Ther 2017; 10: 2791-2804. https://doi.org/10.2147/OTT.S134016
[29] ZHANG J, SHI Z, HUANG J, ZOU X. CSTB Downregulation Promotes Cell Proliferation and Migration and Suppresses Apoptosis in Gastric Cancer SGC-7901 Cell Line. Oncol Res 2016; 24: 487-494. https://doi.org/10.3727/0965 04016X14685034103752

[30] WU YJ, WONG BS, YEA SH, LU CI, WENG SH. Sinularin Induces Apoptosis through Mitochondria Dysfunction and Inactivation of the $\mathrm{pI} 3 \mathrm{~K} / \mathrm{Akt} / \mathrm{mTOR}$ Pathway in Gastric Carcinoma Cells. Mar Drugs 2016; 14: https://doi.org/10.3390/ md14080142

[31] LI NA, WANG W, XU B, GONG H. miR-196b regulates gastric cancer cell proliferation and invasion via PI3K/AKT/ mTOR signaling pathway. Oncol Lett 2016; 11: 1745-1749. https://doi.org/10.3892/ol.2016.4141

[32] LI TT, ZHU D, MOU T, GUO Z, PU JL et al. IL-37 induces autophagy in hepatocellular carcinoma cells by inhibiting the PI3K/AKT/mTOR pathway. Mol Immunol 2017; 87: 132-140. https://doi.org/10.1016/j.molimm.2017.04.010

[33] DING L, WANG S, WANG W, LV P, ZHAO D et al. Tanshinone IIA Affects Autophagy and Apoptosis of Glioma Cells by Inhibiting Phosphatidylinositol 3-Kinase/Akt/Mammalian Target of Rapamycin Signaling Pathway. Pharmacology 2017; 99: 188-195. https://doi.org/10.1159/000452340 